\title{
Katarina Marinčič
}

\section{Divini elementa poetae: les lieux et leurs noms dans I'œuvre de jeunesse de Marcel Proust}

Mots-clés : littérature française du XXe siècle, Marcel Proust, œuvre de jeunesse, Les Plaisirs et les Jours, À la recherche du temps perdu, sens de l'espace

DOI: $10.4312 /$ ars.13.2.102-118

\section{Un humble début}

Le premier livre de Marcel Proust paraît en 1896 chez Calmann-Lévy, à compte d'auteur. Illustré par l'aquarelliste Madeleine Lemaire, enrichi de quatre pièces pour piano de Reynaldo Hahn, préfacé par Anatole France, le recueil hétérogène intitulé Les Plaisirs et les Jours est accueilli sans grand enthousiasme. Malgré la somptuosité de l'édition, voire peut-être à cause de cette somptuosité, les débuts littéraires de Proust sont des plus humbles. Dans l'avant-propos, l'écrivain lui-même qualifie son livre de "vaine écume d'une vie agitée, mais qui maintenant se calme " (Les Plaisirs et les Jours, 12). Le ton de la préface est doucement condescendant : "Sans doute il est jeune. Il est jeune de la jeunesse de l'auteur. Mais il est vieux de la vieillesse du monde. » (Ibid., 7)

Selon une rumeur assez répandue, la préface aux Plaisirs et les Jours ne serait pas écrite par Anatole France mais par sa muse, Mme Arman de Caillavet, amie et protectrice du jeune écrivain1. Quoi qu'il en soit, même ses amis et ses protecteurs semblent le considérer comme un enfant qui passera directement de l'enfance à la vieillesse. (N’est ce pas souvent le cas avec les enfants précoces?)

[...] je m’étais fait de vous une image d'après quelques rencontres dans « le monde ", qui remontent à près de vingt ans. Pour moi, vous étiez resté celui qui fréquente chez Mme X... et Z..., celui qui écrit dans Le Figaro... Je vous croyais - vous l'avouerai-je? - « du côté de chez Verdurin ». (Gide, 9-10)

En janvier 1914, après avoir lu le roman Du côté de chez Swann (publié en 1913 chez Grasset, de nouveau à compte d'auteur), André Gide adresse à Proust une lettre pleine d'admiration et de regret: « Le refus de ce livre restera la plus grave erreur de

1 «Par doute de sa propre valeur et besoin de se sentir éapaulé, Marcel avait fait demander par Madame de Caillavet une préface à Anatole France (et, pour être sûr que celui-ci acceptât, L'Égérie l'avait en partie écrite elle-même [...].» (Maurois, 81) 
la N.R.F. - et (car j’ai cette honte d'en être beaucoup responsable) l'un des regrets, des remords les plus cuisants de ma vie. » (Ibid., 9)

Le regret est compréhensible, mais l'erreur l'est aussi. Gide a seulement deux ans de plus que Proust, il n'a aucune raison pour adopter l'attitude du maître bienveillant envers quelqu'un qu'il considère, à bon droit, comme un dilettante. Dès qu'il ouvre le manuscrit (ce qu'il fait « d'une main distraite»), tous ses préjugés sont confirmés: « Un snob, un mondain amateur, quelque chose d'on ne peut plus fâcheux pour notre revue.» (Ibid.)

Au cours de sa maturité, pendant la période la plus créative de sa vie, Marcel Proust séloigne de la société mondaine. Il lui arrive, semble-t-il, comme à son Elstir: " la pratique de la solitude lui en donne l'amour ». (À l'ombre des jeunes filles en fleurs, 484) Pourtant, même dans l'isolement le plus profond, il reste, par ses goûts et par ses intérêts - un mondain et un snob. Le narrateur de la Recherche est précisément quelqu’un qui «fréquente chez Mme X... et Z... et écrit dans le Figaro ».

Je fermais les yeux en attendant le jour. Je pensai à cet article que j’avais envoyé il y a longtemps déjà au Figaro. J’avais même corrigé les épreuves. Tous les matins, en ouvrant le journal, jespérais le trouver. Depuis plusieurs jours, j'avais cessé d'espérer et je me demandais si on les refusait tous ainsi. (Contre Sainte-Beuve, 84)

Un lecteur assidu de la Recherche sera sans doute impressionné par la lucidité autoironique de certaines pages du recueil posthume Contre Sainte-Beuve. De même, il n'est pas difficile de reconnaître, à postériori, des motifs, voire des leitmotivs proustiens dans Les Plaisirs et les Jours. Du recueil de jeunesse à la grande œuvre de maturité, les thèmes en eux-mêmes nont pas changé : le temps et la mémoire, l'amour et la jalousie, la beauté des femmes et la beauté des fleurs, les bijoux et les toilettes des grandes dames, les pédigrées aristocratiques, le snobisme (à la fois critiqué et partagé), les effets magiques de la musique, la déception immanquablement apportée par la réalisation de nos désirs, l'angoisse du réveil, les troubles de la sensualité, la douce et opprimante présence de Maman...

C'est pour cette raison que même les contemporains les plus lucides ont pu initialement se tromper sur Proust. Les ressemblances thématiques sont faciles à saisir parce que superficielles. La différence entre lécriture du jeune dilettante et lécriture proustienne à son apogée est d’ordre avant tout stylistique - mais dans le sens le plus large du terme. Dans le cas de Proust, changer de style signifie changer d'attitude, de ton, de perspective, d’optique, de vision du monde. Pour citer encore une fois André Gide : «Lorsque nous lisons Proust, nous commençons de percevoir brusquement du détail où ne nous apparaissait jusqu’alors qu'une masse ». (Gide, 102) 
Plus qu'une capacité physique, ce que nous appelons l’œil pour le détail est un trait de caractère. Dans les écrits du jeune Proust, l'habitude d'attacher de l'importance aux choses apparemment insignifiantes se réduit à un tic psychologique. ${ }^{2}$ Chez l'auteur de la Recherche, nous ressentons cette habitude comme une prise de position morale. «Et soudain l’œuvre de Proust, si désintéressée, si gratuite, nous apparaît plus profitable et de plus grand secours que tant d'œuvres dont l'utilité seule est le but. » (Ibid., 103)

« Malgré tant de signes, tant d'erreurs, il avait cru pendant des années appartenir au monde, » écrit un des meilleurs biographes de Proust. (Citati, 225-226) À la fin, pourtant, l'auteur de la Recherche «ne vivait que pour son livre, qui continuait à bouger en lui, à changer de place, à réclamer sans cesse la construction de nouvelles ailes, de tours, d'arcs, d'architraves, ou de détails que personne, peut-être, ne remarquerait, comme personne ne remarque cet ange ou ce monstre sculpté au sommet de la cathédrale d'Amiens et de Chartres. » (Ibid.)

Le but du présent article n'est pas de retracer cette métamorphose, inexplicable sans l'arrière-plan biographique (comme le démontre, entre autres, la magnifique étude de Citati). Nous nous limitons à souligner quelques particularités dans les textes de jeunesse de Proust : particularités qui nous aident peut-être à discerner, au delà des ressemblances thématiques banales et trompeuses, des germes de lécriture proustienne dans lécriture tâtonnante du mondain amateur. Même dans les parties les moins réussies et les plus insignifiantes du recueil Les Plaisirs et les Jours, on peut remarquer une préoccupation que Proust gardera jusquà la dernière page de son grand cycle : le besoin de sorienter dans l'espace - pour pouvoir se placer dans le temps :

[...] il ne me semblait pas que jaurais encore la force de maintenir longtemps attaché à moi ce passé qui descendait déjà si loin. Du moins, si elle métait laissée assez longtemps pour accomplir mon œuvre, ne manquerais-je pas d'abord d'y décrire les hommes (cela dût-il les faire ressembler à des êtres monstrueux) comme occupant une place si considérable, à côté de celle si restreinte qui leur est réservée dans l'espace, une place au contraire prolongée sans mesure - puisqu'ils touchent simultanément, comme des géants plongés dans les années, à des époques si distantes, entre lesquelles tant de jours sont venus se placer - dans le Temps. (Le temps retrouvé, 442)

2 Bien que le jeune Proust partage les vues morales d'une partie de sa generation ; cf. Rosengarten, 119-120: « Proust's ability to reconcile opposing views on many issues in the search for higher and higher levels of philosophical synthesis mark him as basically an eclectic thinker with little if any tolerance for hard-and-fast, absolute positions. [...] Nevertheless, despite this characteristic 'inbetweenness', I think it is fair to say that Proust shared three interrelated values with many writers and intellectuals coming of age in the 1880s and 1890s. These were elitism, individualism, and spiritualism.» 


\section{La métamorphose du temps en espace}

Il est singulier que celui dont on a si souvent voulu faire un disciple de Bergson, ait pris, probablement sans le savoir, une position diamétralement contraire. Si la pensée de Bergson dénonce et rejette la métamorphose du temps en espace, Proust non seulement s'en accommode mais s'y installe, la pousse à l'extrême et en fait finalement un des principes de son art. (Poulet, 9-10)3

Chez Proust, faire l'effort de se souvenir signifie quelque chose de concret et défini, constate Georges Poulet : "Cette tâche porte un nom. Elle s'appelle localisation. Or, de même que l'esprit localise l'image remémorée dans la durée, il la localise dans l'espace. » (Ibid., 26)

La lecture des textes de jeunesse ne nous fait pas nécessairement adhérer à l'interprétation de Poulet selon laquelle « retrouver le lieu perdu » serait pour Proust « sinon la même chose, au moins quelque chose de très semblable au fait de retrouver le temps perdu ». (Ibid.) Il est pourtant indéniable que le jeune écrivain, comme plus tard l'auteur de la Recherche, attache une grande importance à la topographie ${ }^{4}$. De même, la prose juvénile abonde en exemples des déplacements métonymiques considérés comme un des traits les plus distinctifs de l'écriture proustienne ${ }^{5}$ : exemples d'autant plus illustratifs parce que simples et naifs.

Pour le jeune Proust, le futur est un lieu qui apparaît à l'horizon ; le passé, un lieu duquel on séloigne. (Le présent, inutile à dire, est un lieu décevant.)

3 L'idée reprise et développée par Giles Deleuze : «De toute façon, selon la formule bergsonienne, le temps signifie que tout n'est pas donné : le Tout n'est pas donnable. Ce qui veut dire non pas que le Tout 'se fait'dans une autre dimension qui serait précisement temporelle, comme l'entend Bergson ou comme l'entendent pour leur compte les dialecticiens partisans d'un processus de totalisation. Mais parce que le temps, ultime interprète, ultime interpréter, à l'étrange pouvoir d'affirmer simultanément des morceaux qui ne font pas un tout dans l'espace, pas plus qui'ils n'en forment un par succession dans le temps. Le temps est exactement la transversale de tous les espaces possibles, y compris des espaces de temps. » (Deleuze, 157) Cf. Nabokov, 208: « [...] Proustian ideas are colored editions of the Bergsonian thought.»

4 «Ce n'est pas seulement certaine période de son enfance, que l'être proustien voit sortir de sa tasse de thé; c'est aussi une chambre, une église, une ville, un ensemble topographique solide, qui nère plus, qui ne vacille plus. » (Poulet, 27)

5 «Il peut sembler abusif drappeler « métonymie », comme pour le plaisir d’une symétrie factice, cette solidarité des souvenirs qui ne comporte aucun effet de substitution, et qui donc ne peut à aucun titre entrer dans la catégorie des tropes étudiés par la rhétorique. Il suffirait sans doute de répondre que c'est la nature du rapport sémantique qui est en cause ici et non la forme de la figure, et de rappeler que Proust lui-même a donné l'exemple d'un tel abus en baptisant métaphore une figure qui n'est chez lui le plus souvent qu'une comparaison explicite et sans substitution [...]. Enfin, le phénomène de déplacement métonymique, bien connu de la psychanalyse, joue parfois un rôle important dans la thématique même du récit proustien. » (Genette, 58-59) Cf. aussi A l'ombre des jeunes filles en fleurs, 492: « Naturellement, ce qu'il avait dans son atelier, ce nétaient guère que des marines prises ici, a Balbec. Mais j'y pouvais discerner que le charme de chacune consistait en une sorte de métamorphose des choses représentées, analogue à celle qu’en poésie on nomme métaphore [...]. » 
À peine une heure à venir nous devient-elle le présent qu'elle se dépouille de ses charmes, pour les retrouver, il est vrai, si notre âme est un peu vaste et en perspectives bien ménagées, quand nous l’aurons laissée loin derrière nous, sur les routes de la mémoire. Ainsi le village poétique vers lequel nous hâtions le trot de nos espoirs impatients et de nos juments fatiguées exhale de nouveau, quand on a dépassé la colline, ces harmonies voilées, dont la vulgarité de ses rues, le disparate de ses maisons, si rapprochées et fondues à l'horizon, l'évanouissement du brouillard bleu qui semblait le pénétrer, ont si mal tenu les vagues promesses. (Les Plaisirs et les Jours, 219-220)

Un autre exemple : le retour en arrière (flashback) vécu par le protagoniste de la nouvelle La mort de Baldassare Silvande. Les mémoires qui envahissent la conscience du mourant pour s'y amalgamer en une seule mémoire sont éveillées (plus exactement : apportées) par un son qui vient " d'un village extrêmement éloigné » :

Tout à coup il entendit un petit bruit argentin, imperceptible et profond comme un battement de cœur. C'était le son des cloches d'un village extrêmement éloigné, qui, par la grâce de l'air si limpide ce soir-là et de la brise propice, avait traversé bien des lieues de plaines et de rivières avant d’arriver jusquà lui pour être recueilli par son oreille fidèle. (Ibid., 42)

Dans un autre récit des dernières heures, l'héroïne de la lugubre Confession d'une jeune fille attend impatiemment la mort après sêtre tiré une balle dans la poitrine. Tourmentée par les remords, elle forme le désir de retourner, pour y retrouver son innocence perdue - non pas au temps de son enfance ou de sa première jeunesse, mais dans un parc, à un « lieu plein de sa mère ».

Si je nétais pas si faible, si javais assez de volonté pour me lever, pour partir, je voudrais aller mourir aux Oublis, dans le parc où j’ai passé tous mes étés jusquà quinze ans. Nul lieu nest plus plein de ma mère, tant sa présence, et son absence plus encore, l'imprégnèrent de sa personne. (Ibid., 138)

\section{Les portraits, les arrière-plans et les cadres}

Proust dédie Les Plaisirs et les Jours à la mémoire de son ami Willie Heath, mort tragiquement jeune en 1893. Dans l'avant-propos, il évoque avec beaucoup d'émotion lélégance physique et morale du dédicataire.

C'est au Bois que je vous retrouvais souvent le matin, mayant aperçu et mattendant sous les arbres, debout, mais reposé, semblable à un de ces seigneurs qu’a peints Van Dyck et dont vous aviez l'élégance pensive. Leur élégance, en effet, comme la vôtre, réside moins dans les vêtements que dans le 
corps, et leur corps lui-même semble l'avoir reçue et continuer sans cesse à la recevoir de leur âme : c'est une élégance morale. (Ibid., 10)

La valeur biographique de ces réminiscences dépasse leur qualité littéraire. Il est pourtant intéressant de remarquer que, dans un texte qui n'est, au fond, qu'une déclaration d'amour à peine voilée, Marcel semble mettre une emphase particulière sur le lieu de ses rencontres avec Willie: «C'est au Bois que je vous retrouvais souvent le matin ». Les feuillages en arrière-plan sont essentiels, le cadre est presque aussi important que le portrait.

Dans la Mélancolique villégiature de Mme de Breyves, l'héroïne est même réduite à se contenter de l'arrière-plan. Éperdument amoureuse d'un homme qui - pour employer les célèbres mots de Swann - «ne lui plaît pas, n'est pas son genre ", elle se réfugie d'abord dans la musique. La musique lui apporte, comme à Swann, autant de souffrance que de bonheur:

Une phrase des Maîtres chanteurs entendue à la soirée de la princesse d'A... avait le don de lui évoquer M. de Laléande avec le plus de précision [...]. Elle en avait fait sans le vouloir le véritable leitmotiv de M. de Laléande, et, l'entendant un jour à Trouville dans un concert, elle fondit en larmes. De temps en temps, pas trop souvent pour ne pas se blaser, elle s'enfermait dans sa chambre, où elle avait fait transporter le piano et se mettait à la jouer en fermant les yeux pour mieux le voir, cétait sa seule joie grisante avec des fins désenchantées, l'opium dont elle ne pouvait se passer. (Ibid., 118)

À cette drogue, elle en ajoute une autre. Sachant que l'homme insignifiant qu'elle adore passe ses vacances à Biarritz, elle devient obsédée par ce lieu:

Elle en est arrivée, elle qui détestait Biarritz, à trouver à tout ce qui touche à cette ville un charme douloureux et troublant. Elle s'inquiète des gens qui y sont, qui le verront peut être sans le savoir, qui vivront peut-être avec lui sans en jouir. Pour ceux-là elle est sans rancune, et sans oser leur donner de commissions, elle les interroge sans cesse, sétonnant parfois qu'on l'entende tant parler à l'entour de son secret sans que personne l'ait découvert. Une grande photographie de Biarritz est un des seuls ornements de sa chambre. Elle prête à l'un des promeneurs qu'on y voit sans le distinguer les traits de M. de Laléande. (Ibid., 123)

Un lecteur familier de la psychologie proustienne ne serait pas surpris d'apprendre que Mme de Breyves prononce dans son sommeil le nom de - Biarritz. 


\section{La force évocatrice des noms de lieux}

Pour le narrateur de la Recherche, les noms des lieux possèdent une force évocatrice:

Je n'eus besoin pour les faire renaître que de prononcer ces noms: Balbec, Venise, Florence, dans l'intérieur desquels avait fini par s'accumuler le désir que mavaient inspiré les lieux qu'ils désignaient. (Du côté de chez Swann, 449)

Par une métonymie au second degré, les noms de lieux aussi obtiennent une dimension temporelle. Le nom d'un lieu lointain est plein de désir, donc plein de futur. À la fois, le charme même de ce nom est la cause des déceptions à venir, de ce présent au futur démoralisant qui nous envoyera chercher le bonheur dans le passé, « sur les routes de la mémoire».

Mais si ces noms absorbèrent à tout jamais l'image que j’avais de ces villes, ce ne fut qu'en la transformant, qu'en soumettant sa réapparition en moi à leurs lois propres ; ils eurent ainsi la conséquence de la rendre plus belle, mais aussi plus différente de ce que les villes de Normandie ou de Toscane pouvaient être en réalité, et, en accroissant les joies arbitraires de mon imagination, d'aggraver la déception future de mes voyages. (Ibid., 450)

Les textes de jeunesse, notamment les récits à la première personne, sont imprégnés de cette psychologie du voyage. (Si les protagonistes de la rêverie intitulé Présence réelle ne sont pas déçus par les lieux qu'ils visitent, c'est parce que leur liaison, et par conséquent leur voyage, est imaginaire.)

Nous nous sommes aimés dans un village perdu d'Engadine au nom deux fois doux : le rêve des sonorités allemandes s'y mourait dans la volupté des syllabes italiennes. [...] - un jour (nous n'avions ni l'un ni l'autre jamais vu l'Italie), nous restâmes comme éblouis de ce mot qu'on nous dit de l'Apron : « De là on voit jusqu'en Italie. " Nous partîmes pour l'Apron, imaginant que, dans le spectacle étendu devant le pic, là où commencerait l'Italie, le paysage réel et dur cesserait brusquement et que s'ouvrirait dans un fond de rêve une vallée toute bleue. En route, nous nous rappelâmes qu'une frontière ne change pas le sol et que si même il changeait ce serait trop insensiblement pour que nous puissions le remarquer ainsi, tout d'un coup. Un peu déçus nous riions pourtant d'avoir été si petits enfants tout à l'heure.

Mais en arrivant au sommet, nous restâmes éblouis. Notre enfantine imagination était devant nos yeux réalisée. [...] Les noms nétaient plus les mêmes, aussitôt s'harmonisaient avec cette suavité nouvelle. On nous montrait le lac de Poschiavo, le pizzo di Verone, le val de Viola. [...] Je sentis 
alors vraiment à fond la tristesse de ne t’avoir pas avec moi sous tes matérielles espèces, autrement que sous la robe de mon regret, en la réalité de mon désir. (Les Plaisirs et les Jours, 213-214)

En fin du compte, même les amours non réalisés nous désillusionnent - et, même dans ce cas, les noms de lieux gardent leur force évocatrice. Plutôt que de perdre leur pouvoir, ils l'usurpent, s'appropriant un charme qui ne leur appartient que par association.

La satiété est venue avant la possession. [...] Ta vue ne garde pour moi qu'un charme, celui de me rappeler tout à coup ces noms d'une douceur étrange, allemande et italienne : Sils Maria, Silva Plana, Crestalta, Samaden, Celerina, Juliers, val de Viola. (Ibid., 217)

\section{Souvenir des chambres}

Selon Roland Barthes, "toute La Recherche du temps perdu se donne comme un ébranlement par ondes élargissantes du souvenir des chambres ». (Barthes, 304) En effet, certaines parties du premier livre de Proust apparaissent comme une illustration caricaturale de cette constatation. Ainsi, le petit texte prétentieux intitulé Scénario souvre avec une phrase qui, pour le dire gentiment, ne suscite pas de grandes attentes: "Honoré est assis dans sa chambre. » (Les Plaisirs et les Jours, 78) Ensuite, le protagoniste " se lève et se regarde dans la glace ", puis il s'engage dans une conversation avec sa Cravate, sa Plume, « des Roses, des Orchidées des Hortensias, des Cheveux-de-Vénus, des Ancolies, qui remplissent la chambre ", ses Livres et, finalement, après un court échange avec sa Bonne Fée, avec la Petite Pendule de Saxe. (Ibid., 78-80)

Dans le texte intitulé Éventail, un autre exercice d'écolier que Marcel inclut dans son livre de débutant, le protagoniste-narrateur fait à une grande dame le cadeau d'un éventail sur lequel il a peint, comme il dit, « les formes vaines et charmantes qui peuplèrent votre salon [...] à jamais fermé maintenant ». (Ibid., 81)

Les lustres, dont toutes les branches portent de grandes fleurs pâles, éclairent des objets d'art de tous les temps et de tous les pays. Je pensais à l'esprit de notre temps en promenant avec mon pinceau les regards curieux de ces lustres sur la diversité de vos bibelots. (Ibid., 82)

Pourtant, dans ce même salon encombré de bibelots, ou bien sur cette même peinture, une petite scène nous indique que, pour les hommes et les femmes vraiment raffinés, une chambre n'est que le point de départ:

Je n’ai pas craint de vous figurer sur le canapé rose. T... y est assis auprès de vous. Il vous décrit sa nouvelle chambre savamment goudronnée pour lui suggérer 
les sensations d'un voyage en mer, vous dévoile toutes les quintessences de sa toilette et de son ameublement.

Votre sourire dédaigneux témoigne que vous prisez peu cette imagination infirme à qui une chambre nue ne suffit pas pour y faire passer toutes les visions de l'univers, et qui conçoit l'art et la beauté d'une façon si pitoyablement matérielle. (Ibid., 84)

Dans La mort de Baldassare Silvande, la sublime imagination d'un mourant n'a même pas besoin d'une chambre nue, elle finit par se suffire à elle-même. Pendant sa longue maladie, Baldassare aime se tenir « dans une immense pièce ronde entièrement vitrée ».

En entrant en voyait en face de soi la mer, et, en tournant la tête, des pelouses, des pâtrourages et des bois ; au fond de la pièce, il y avait deux chats, des roses, des pavots et beaucoup d'instruments de musique. (Ibid., 20)

Vers la fin de sa vie, quand il n'a plus de force pour se lever, le malade fait porter son lit dans la rotonde. La beauté de la vue (ou plutôt des vues) augmente l'amertume de ses regrets.

Lair était si doux qu’on ouvrit les fenêtres qui regardaient la mer sans la voir, et à cause du vent trop vif on laissa fermées celles d’en face, devant qui sétendaient les pâturages et les bois. Baldassare fit traîner son lit près des fenêtres ouvertes. Un bateau, mené à la mer par les marins qui sur la jetée tiraient la corde, partait. Un beau mousse d'une quinzaine d'années se penchait à l'avant, tout au bord ; à chaque vague, un croyait qu'il allait tomber dans l'eau, mais il se tenait ferme sur ses jambes solides. Il tendait le filet pour ramener le poisson et tenait une pipe chaude entre ses lèvres salées par le vent. Et le même vent qui enflait la voile venait rafraîchir les joues de Baldassare et fit voler un papier dans la chambre. Il détourna la tête pour ne plus voir cette image heureuse des plaisirs qu'il avait passionnément aimés et qu'il ne goûterait plus. (Ibid., 42)

Devenant de plus en plus semblable aux fenêtres qui " regardent sans voir $»^{6}$, Baldassare est de moins en moins libre de choisir sa perspective.

Baldassare fit fermer les fenêtres de ce côté de la rotonde et ouvrir celles qui donnaient sur les pâturages et les bois. Il regarda les champs, mais il entendait encore le cri d'adieu poussé sur le trois-mâts, et il voyait le mousse, la pipe entre les dents, sur le trois-mâts, et il voyait le mousse, la pipe entre les dents, qui tendait ses filets. (Ibid.)

6 De même que le peintre d'éventail, dans le texte précédent, « promène avec son pinceau le regard curieux des lustres ». 
Or, cette perte de liberté est une bénédiction déguisée: il suffit, pour mourir paisiblement, d’avoir été libéré de la nécessité de choisir:

Baldassare reposait, les yeux fermés [...]. Il revit sa mère quand elle l’embrassait en rentrant, puis quand elle le couchait le soir et réchauffait ses pieds dans ses mains, restant près de lui s'il ne pouvait s'endormir. (Ibid., 43)

\section{Le sommeil et le réveil}

«La chambre proustienne est toujours, forcément, celle du coucher: l'insomnie de l'adulte se nourrit des angoisses de l'enfant, ressuscitées par la sensation précise de loreiller, de la veilleuse. » (Wallenstein, 63)

Indubitablement, il existe un lien étroit entre le sommeil, l'insomnie et le lieu où le personnage proustien essaye, avec un succès variable, de prendre repos. Les chambres déclenchent des mémoires, provoquent des états d’âme particuliers, affectent la capacité de s'endormir du narrateur et de maint protagoniste de la Recherche. Néanmoins, c'est l'insomnie et non pas le lieu en soi qui provoque la souffrance. Pour le dire d'une façon naïve et simpliste : le sommeil en soi est plus important que la chambre, les conséquences sont plus importantes que les catalyseurs. Exemple illustratif : dans ses variations sur des thèmes de Proust, surtout dans le projet inabouti intitulé Lieux où jai dormi, Georges Perec attire notre attention sur une différence essentielle entre sa propre vision du monde et la vision proustienne. La tâche que s'impose Perec est de garder la mémoire des lieux. ${ }^{7}$ Or, selon la sensibilité proustienne, ce sont les lieux qui gardent les mémoires.

Quand le personnage proustien reconnaît sa chambre au moment de se réveiller, l'apaisement (ou bien le désenchantement) qui l'envahit n’est pas celui d'avoir retrouvé ses meubles et ses bibelots, mais celui d'avoir retrouvé sa place dans l'univers. L'effort de se réveiller est donc semblable à l'effort de se souvenir. Dans les deux cas, l'essentiel semble être la localisation spatiale. En se réveillant, le personnage proustien ne se demande pas quelle heure il est, ou bien quel temps il fait. Il ressent un besoin profond et urgent de savoir où il est : comme si, en ne le sachant pas, il serait en danger de se perdre, dans tous les sens possibles du mot. C'est la peur de se perdre plus précisément : la peur d'oublier cette peur dans un moment d'insouciance - qui provoque l'insomnie. Quant à la zone qui se situe dans le temps entre le sommeil et le réveil, cet espace éminemment proustien peut être soit un lieu de bonheur soit un lieu d'angoisse.

7 Voir à ce propos le bel article From Lieux to Life de Tom Emerson: « If Perec is so concerned that his characters are constructed by space and things, it is because the characters, in themselves, are incapable of remembrance. Space is the locus of memory (and history) and it must, therefore, be protected in order to prevent erasure. It must be described so that it can survive. » (Emerson, 92) 
Au moment de s'endormir, au moment inverse et correspondant du réveil, dans l'espèce de clair obscur où la conscience est moins armée pour résister aux phénomènes qui la troublent, il arrive donc parfois au personnage proustien de voir l'espace se scinder, se dédoubler, perdre sa simplicité et son immobilité apparentes. Et il se peut que cette expérience ait pour effet chez celui qui léprouve, un vertigineux bonheur. Mais la plupart du temps, la découverte du caractère instable des lieux lui inspire, bien au contraire, un sentiment d'appréhension et même d'horreur. (Poulet, 18)

Il ne faut pourtant pas que ce soit à mi chemin entre le sommeil et la veille : ce même " bonheur vertigineux " peut être ressenti par un homme pleinement éveillé, à condition qu'il perde la conscience de l'espace réel - comme le prouve le célèbre épisode des pavés inégaux évoquée par Samuel Beckett (un auteur d'ailleurs plus sensible au côté sombre qu'au côté joyeux de l'écriture proustienne):

Crossing the courtyard he stumbles on the cobbles. His surroundings vanish, wattmen, stables, carriages, guests, the entire reality of the place in its hour, his anxiety and doubts as to the reality of life and art disappear, he is stunned by waves of rapture, saturated in that same felicity that had irrigated so sparingly the desolation of his life. Drabness is obliterated in an intolerable brightness. And suddenly Venice emerges from the series of forgotten days [...]. (Beckett, 52)

Et, en définitive : la chambre n'est pas le seul lieu où l'on peut s'endormir. Dans la Sonate clair de lune, le protagoniste-narrateur s'assoupit en plein air. Son réveil presque aussi onirique que le sommeil - est d'une ambiguïté légèrement inquiétante. Rejoint par une jeune femme, il se sent entrainé avec elle dans le «doux désespoir irrésistible " de la lune : "Comme nous, elle pleurait, et comme nous faisons presque toujours, elle pleurait sans savoir pourquoi [...]»(Les Plaisirs et les Jours, 190)

Avant de séveiller dans le sens usuel du mot, le jeune homme connaît la joie triomphale de séveiller au rêve. Pas un rêve quelconque, mais un rêve nervalien, un rêve qui est « une seconde vie ${ }^{8}$ ».

Je me couchai sur le gazon, la figure tournée vers le ciel sombre ; bercé par le bruit de la mer, que j’entendais derrière moi, sans bien la distinguer dans l’obscurité, je ne tardai pas à m’assoupir. Bientôt je rêvai que devant moi, le

8 «Le rêve est une seconde vie. [...] C’est un souterrain vague qui séclaire peu à peu, et où se dégagent de l'ombre et de la nuit les pâles figures gravement immobiles qui habitent le séjour des limbes. Puis le tableau se forme, une clarté nouvelle illumine et fait jouer ces apparitions bizarres : - le monde des Esprits s'ouvre pour nous. » (Aurélia, 17). Cf. Contre Sainte-Beuve, 160 : « [...] Gérard de Nerval a trouvé les moyens de ne faire que peindre et de donner à son tableau les couleurs de son rêve. Peutêtre y a-t-il encore un peu trop d'intelligence dans sa nouvelle. " Au sujet des affinités entre Proust et Nerval voir Hong Kuo-Yung. 
coucher du soleil éclairait au loin le sable et la mer. Le crépuscule tombait, et il me semblait que cétait un coucher de soleil et un crépuscule comme tous les crépuscules et tous les couchers de soleil. Mais on vint m’apporter une lettre, je voulus la lire et je ne pus rien distinguer. Alors seulement je m’aperçus que malgré cette impression de lumière intense et épandue, il faisait très obscur. Ce coucher de soleil était extraordinairement pâle, lumineux sans clarté, et sur le sable magiquement éclairé s’amassaient tant de ténèbres qu'un effort pénible métait nécessaire pour reconnaître un coquillage. Dans ce crépuscule spécial aux rêves, c'était comme le coucher d'un soleil malade et décoloré, sur une grève polaire. Mes chagrins sétaient soudain dissipés [...]. La contradiction de ce resplendissement obscur, le miracle de cette trêve enchantée à mes maux ne m'inspirait aucune défiance, aucune peur, mais j’étais enveloppé, baigné, noyé d'une douceur croissante dont l'intensité délicieuse finit par me réveiller. J'ouvris les yeux. Splendide et blême, mon rêve sétendait autour de moi. Le mur auquel je métais adossé pour dormir était en pleine lumière, et l'ombre de son lierre s'y allongeait aussi vive qu'à quatre heures de l'aprèsmidi. Le feuillage d'un peuplier de Hollande retourné par un souffle insensible étincelait. On voyait des vagues et des voiles blanches sur la mer, le ciel était clair, la lune s'était levée. Par moments, de légers nuages passaient sur elle, mais ils se coloraient alors de nuances bleues dont la pâleur était profonde comme la gelée d'une méduse ou le cœur d'une opale. La clarté pourtant qui brillait partout, mes yeux ne la pouvaient saisir nulle part. Sur l'herbe même, qui resplendissait jusqu'au mirage, persistait l'obscurité. Les bois, un fossé, étaient absolument noirs. Tout d'un coup, un bruit léger séveilla longuement comme une inquiétude, rapidement grandit, sembla rouler sur le bois. C'était le frisson des feuilles froissées par la brise. Une à une je les entendais déferler comme des vagues sur le vaste silence de la nuit tout entière. Puis ce bruit même décrut et seéteignit. Dans létroite prairie allongée devant moi entre les deux épaisses avenues de chênes, semblait couler un fleuve de clarté, contenu par ces deux quais d’ombre. La lumière de la lune, en évoquant la maison du garde, les feuillages, une voile, de la nuit où ils étaient anéantis, ne les avait pas réveillés. Dans ce silence de sommeil, elle néclairait que le vague fantôme de leur forme, sans qu'on pût distinguer les contours qui me les rendaient pendant le jour si réels, qui mopprimaient de la certitude de leur présence, et de la perpétuité de leur voisinage banal. La maison sans porte, le feuillage sans tronc, presque sans feuilles, la voile sans barque, semblaient, au lieu d'une réalité cruellement indéniable et monotonement habituelle, le rêve étrange, inconsistant et lumineux des arbres endormis qui plongeaient dans l’obscurité. Jamais, en effet, les bois n'avaient dormi si profondément, on 
sentait que la lune en avait profité pour mener sans bruit dans le ciel et dans la mer cette grande fête pâle et douce. Ma tristesse avait disparu. (Les Plaisirs et les Jours, 187-189)

Comme dans une synthèse prophétique, la vision du jeune dormeur nous fait entrevoir les deux sources de l'art proustien : la sensibilité de « quelqu'un dont le regard est infiniment plus subtil et plus attentif que le notre »(Gide, 103) et la force titanique d'un artiste prêt à distordre la réalité, dissoudre l'espace, abolir la séparation entre les éléments ${ }^{9}$ - pour arriver à la vérité. Un écrivain qui - pareil au grand peintre Elster - ne vit que pour «les rares moments où l'on voit la nature telle quelle est, poétiquement ». (À lombre des jeunes filles en fleurs, 492)

\section{Bibliographie}

Barthes, R., La Préparation du roman I et II. Cours au Collège de France (1978-1979 et 1979-1980), Paris 2003.

Becket, S., Proust, New York 1978.

Citati, P., La colombe poignardée : Proust et la Recherche, Paris 1997.

Deleuze, G., Proust et les signes, Paris 1964.

Emerson, T., From Lieux to Life..., AA Files, no. 45/46, 2001, pp. 92-97.

Genette, G., Figures III, Paris 1972.

Gide, A., Proust, M., Autour de La Recherche: lettres, Bruxelles 1988.

Hong, Kuo-Yung, Proust et Nerval. Essai sur les lois mystérieuses de l'écriture, Paris 2006.

Maurois, A., À la recherche de Marcel Proust, Paris 1985.

Nabokov, V., Lectures on Literature, New York 1980.

Nerval, G. de, Aurélia, Paris 1958.

Poulet, G., L'espace proustien, Paris 1982.

Proust, M., Les Plaisirs et les Jours, Paris 1980.

Proust, M., Contre Sainte-Beuve, Paris 1954.

Proust, M., Du côté de chez Swann, Paris 1954.

Proust, M., À l’ombre des jeunes filles en fleurs, Paris 1954.

9 « Parfois à ma fenêtre [...] il métait arrivé, grâce à un effet de soleil, de prendre une partie plus sombre de la mer pour une côte éloignée, ou de regarder avec joie une zone bleue et fluide sans savoir si elle appartenait à la mer ou au ciel. Bien vite mon intelligence rétablissait entre les éléments la séparation que mon impression avait abolie. [...] Mais les rares moments où l'on voit la nature telle qu'elle est, poétiquement, c'était de ceux-là queetait faite l’œuvre d'Elstir. » (À l’ombre des jeunes filles en fleurs, 492) 
Proust, M., Le temps retrouvé, Paris 1954.

Rosengarten, F., The Writing of the Young Marcel Proust (1885-1900): An Ideological Critique, New York 2001.

Wolkenstein, J., L’espace intime de la fiction, in: Poétique de la maison (éd. Lévillain, H.), Paris 2005. 


\section{Katarina Marinčič}

\section{Divini elementa poetae: les lieux et leurs noms dans I'œuvre de jeunesse de Marcel Proust}

Mots-clés : littérature française du XXe siècle, Marcel Proust, œuvre de jeunesse, Les Plaisirs et les Jours, À la recherche du temps perdu, sens de l'espace

Larticle aborde le sens de l'espace dans l'œuvre de jeunesse de Marcel Proust, notamment dans son premier livre Les Plaisirs et les Jours.

Le recueil hétérogène des textes de jeunesse que Proust publie en 1896 à compte d'auteur cimente sa réputation de dilettante et de snob. En ce qui concerne son début littéraire, le jugement des contemporains sur Proust a été confirmé par la postérité. Tout en reconnaissant l'abîme qui sépare Les Plaisirs et les Jours de la Recherche, nous nous proposons de souligner quelques particularités des textes de jeunesse qui nous aident peut-être à discerner, au delà des ressemblances thématiques banales et trompeuses, des germes de l'écriture proustienne dans l'écriture tâtonnante du mondain amateur. Même dans les parties les moins réussies et les plus insignifiantes des Plaisirs et les Jours, on peut remarquer une préoccupation que Proust gardera jusquà la dernière page de son grand cycle: le besoin de s'orienter dans l'espace - pour pouvoir se placer dans le temps. 


\section{Katarina Marinčič}

\section{Divini elementa poetae: kraji in njihova imena $v$ mladostnem delu Marcela Prousta}

Ključne besede: francoska književnost 20. stoletja, Marcel Proust, mladostno delo, Radosti in dnevi, $V$ iskanju izgubljenega časa, občutenje prostora

Članek obravnava občutenje prostora v mladostnem delu Marcela Prousta. Heterogena zbirka Radosti in dnevi, ki jo je pisatelj v samozaložbi izdal leta 1896, je med sodobniki utrdila njegov sloves diletanta, snoba in salonskega leva. Ta sodba je v veliki meri obveljala. Med Proustovim prvim literarnim poskusom in njegovim vélikim delom, ciklom $V$ iskanju izgubljenega časa, tudi s stališča poznejših bralcev in kritikov zeva osupljiv prepad. Vendar ta prepad, kot skušamo pokazati v pričujoči kratki razpravi, ni toliko v spremenjeni tematiki (proustovske teme so v mladostnem delu že močno prisotne) kakor v slogovnih premikih, ki potegnejo za seboj globoke vsebinske spremembe.

Vsaj a posteriori pa lahko $\mathrm{v}$ mladostni prozi že razbiramo nastavke poznejše proustovske optike. Ti so morda še posebej opazni v odnosu, ki ga avtor zbirke Radosti in dnevi vzpostavlja do prostora: $\mathrm{s}$ tem, da časovno oddaljenost ponazarja $\mathrm{z}$ razdaljo $\mathrm{v}$ prostoru; s tem, da poseben pomen pripisuje krajem in evokativnosti njihovih imen; $\mathrm{s}$ pozornostjo, ki jo posveča ozadjem in prizoriščem; ter naposled: z zabrisovanjem prostorskih razmejitev, s pojavom, ki ga pripovedovalec Iskanja izgubljenega časa doživlja na meji med snom in budnostjo, slikar Elstir, največji umetnik med osebami Proustovega cikla, pa si iz njega izpelje umetniški princip. 


\section{Katarina Marinčič}

\section{Divini elementa poetae: Places and their Names in the Youthful Work of Marcel Proust}

Keywords: $20^{\text {th }}$-century French literature, Marcel Proust, Pleasures and Days, In Search of Lost Time, youthful work, sense of space

The paper addresses the sense of space in Marcel Proust's youthful work. The collection of Proust's early texts, published in 1896 at the author's expense under the title Pleasures and Days, cemented his reputation as a dilettante, a snob and a salon lion among his contemporaries. This opinion stood the test of time. Even from the standpoint of later readers and critics, there is a striking gap between Proust's first literary attempt and his great work, the cycle In Search of Lost Time. However, as we are trying to show, this gap is not caused by changes in the subject matter (Proustian themes and motifs are already heavily present in his early work), but in stylistic shifts that entail profound changes of meaning.

At least a posteriori, we can discern some crucial elements of the Proustian vision in his early prose. These may be particularly noticeable in regard to the young Proust's relation to space: his habit of illustrating distance in time with distance in space; the importance that he attaches to places and the evocative power of their names; the attention he pays to backgrounds and venues; and finally, his fascination with the dissolution of spatial boundaries, a phenomenon that the narrator of the Search for Lost Time experiences in the moments between sleep and wakefulness, while the painter Elstir, the greatest artist among Proust's literary characters, derives an artistic principle from it. 